\title{
AN MOS DOSIMETER FOR USE IN SPACE
}

\author{
L. S. August, R. R. Circle, and J. C. Ritter
}

Naval Research Laboratory
Washington, DC 20375

J. S. Tobin

Naval Electronic Systems Command

Washington, DC 20360

\section{ABSTRACT}

This paper describes a four-sensor, integrating dosimeter that is designed for use aboard earth-orbiting satellites. The radiation-detecting sensors utilized are radiation-soft, PMOS transistors. The dosimetric parameter employed is the shift in threshold voltage, $\Delta V_{T}$. This voltage shift is related to the dose absorbed, $D$, in the $\mathrm{SiO}_{2}$ film of the transistor. The relationship between $\Delta V_{T}$ and $D$ is determined with a calibrated Co-60 gamma-ray source. The design of the dosimeter minimizes the differences in charge recombination between the calibrating radiation, Co-60 gamma rays, and the space radiation environment, which consists mostly of energetic electrons and protons. Each of the four transistors that are incorporated into the Spacecraft MOS Dosimeter is beneath a different thickness of aluminum in order to obtain depth-dose information. The circuit that applies the required biases to the transistor gates and also provides the needed analog voltage data to the spacecraft telemetry system consists mostly of radiation-hard operational amplifiers and stable, precision resistors. This circuit has been tested to $150 \mathrm{krad}$ with $7-\mathrm{MeV}$ electrons, and there was no observable degradation in performance.

\section{INTRODUCTION}

The word dosimeter is often used in two senses (first for the entire unit and secondly for the individual sensors). In order to avoid confusion as to the intended meaning, these two uses are clarified immediately in the following discussion. The type of radiation sensor employed in this work is a $p$-channel metal-oxide semiconductor (PMOS) field-effect transistor. Four such transistors are contained in the instrument developed for use aboard spacecraft. The complete package is referred to as the Spacecraft MOS Dosimeter (SMD), and an individual sensor is designated as a PMOS transistor or device. To illustrate better what is being discussed, the first three figures show the two types of PMOS transistors utilized in this work as well as a unit of the SMD.

Figure 1 is a photograph of a PMOS transistor fabricated in the manner described by Adams and Holmes-Siedle (1). These devices are obtained from a microelectronics fabrication facility in the United Kingdom (2) and are referred to as the UK PMOS devices or transistors. The

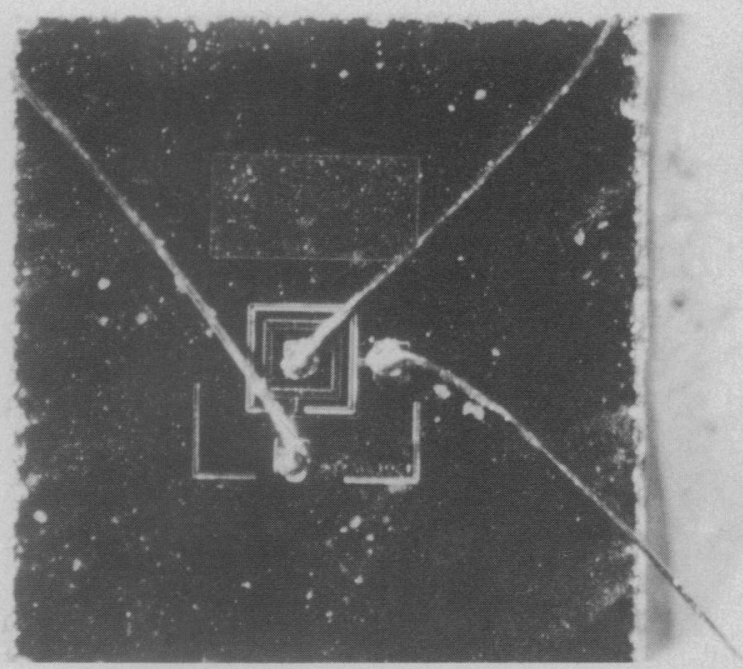

Fig. 1 - UK PMOS device with connections to the transistor. The rectangular structure is an MOS capacitor. gate oxide has a nominal thickness of 0.12 micrometers. Gate oxidation and subsequent annealing are carried out at relatively high temperatures $\left(>1000^{\circ} \mathrm{C}\right)$. The dimensions of the chip, which also contains an MOS capacitor, are $3 \mathrm{~mm}$ by $3 \mathrm{~mm}$, and the overall dimensions of the PMOS transistor itself are 500 micrometers by 500 micrometers. The gate length is 5 micrometers. The chip is hermetically sealed in a TO-5 container when it is to be employed as a sensor aboard a spacecraft.

Figure 2 is a photograph of an RCA CD4007 chip (3). Ordinarily the three CMOS inverters on this chip are used. However, for the dosimetric application, the PMOS transistor test structure is the only component of interest on the chip. Bonding wires are attached to this structure, and the chip is suitably encapsulated in a TO- 5 container. This RCA PMOS device is also processed at high temperatures $\left(>1000^{\circ} \mathrm{C}\right)$, and it has properties very similar to those of the UK PMOS transistor. Some of the data to be discussed are taken with the RCA PMOS devices since they are being evaluated for possible use in the SMD. The oxide thickness of the RCA PMOS transistor has a nominal value of 0.11 micrometers, and this smaller oxide thickness can account for the somewhat lower sensitivity to radiation exhibited by the RCA PMOS devices as compared to those from the UK. The dimensions of the chip are 1.4 $\mathrm{mm}$ by $1.5 \mathrm{~mm}$, and the gate length is 7.6 micrometers

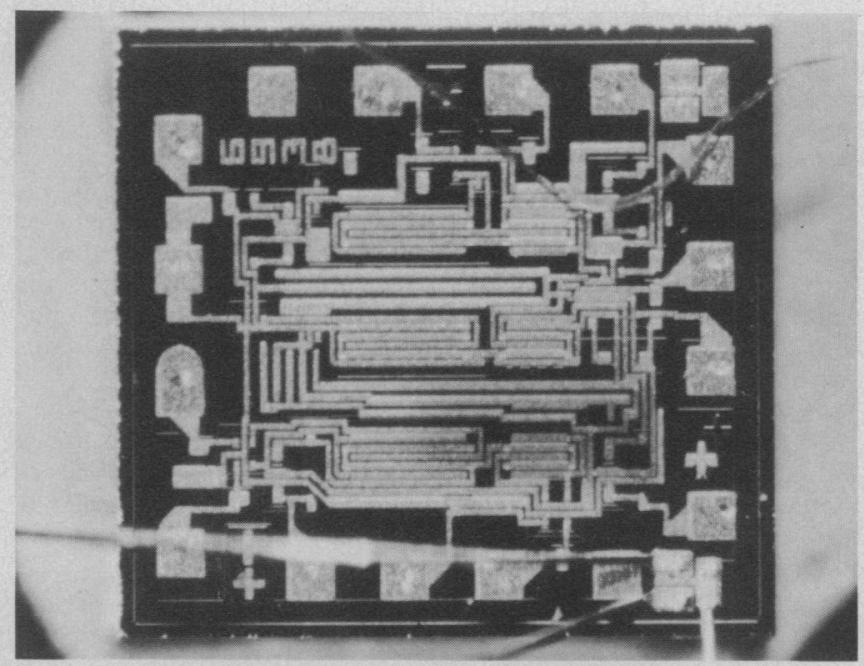

Fig. 2 - RCA CD4007 chip with connecting wires to the PMOS test transistor in the lower right-hand corner.

A unit of the SMD is shown in Fig. 3 with the protective cover removed. Four PMOS transistors are employed with each beneath a different thickness of aluminum in order to obtain depth-dose information. Units of the type shown in the figure have a maximum weight of 187 grams, the average power required is 120 milliwatts, and the dimensions are $7.0 \mathrm{~cm}$ by $7.0 \mathrm{~cm}$ by $4.0 \mathrm{~cm}$. These dimensions do not include the bracket extensions.

Having defined the terms that will be used, introductory comments on the SMD are now in order. Holmes-Siedle (4) first proposed using a PMOS transistor to measure dose aboard a spacecraft. The dosimetric parameter employed to determine the dose, $D$, is the shift in threshold voltage, $\Delta V_{T}$. Since there is not currently a convenient way to reset the threshold voltage level aboard a spacecraft, the total shift in threshold voltage is determined as a function of time and thereby provides a measure of the integrated dose. A calibrated Co-60 gamma-ray source is

U.S. Government work not protected by U.S. copyright. 


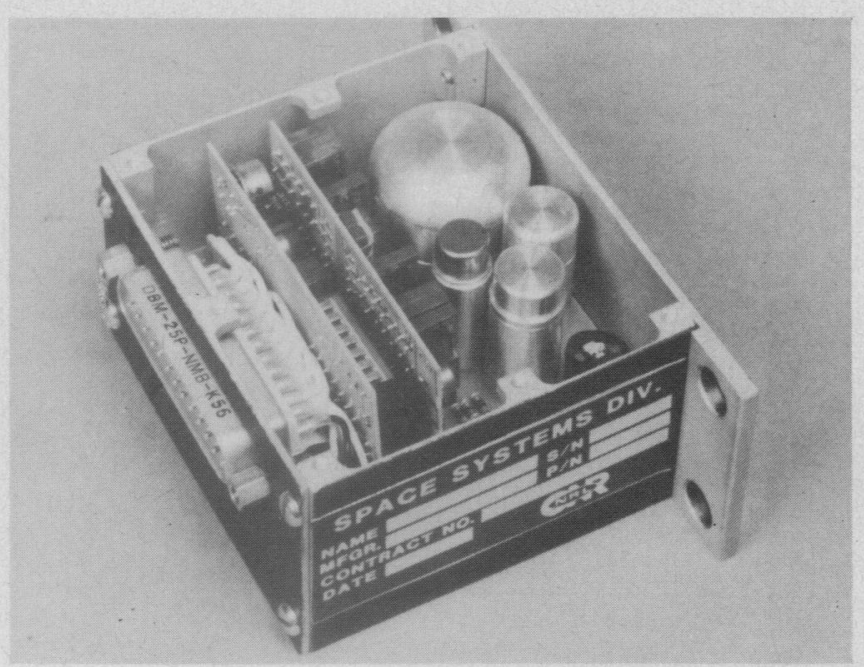

Fig. 3 - The Spacecraft Mos Dosimeter (SMD) with the top protective cover removed. The four PMOS devices are surrounded by different amounts of shielding. The least heavily shielded device is surrounded only by a TO- 5 container, and the most heavily shielded one by $0.9 \mathrm{~cm}$ of aluminum.

employed in determining the relationship between $\Delta V_{T}$ and $D$. The space radiation environment, on the other hand, consists mostly of electrons and protons. Consequently, in the design of the SMD attention is paid to the differences in the response per krad for the radiations involved. These variations in response occur because the radiations studied produce ionization tracks of different densities in the $\mathrm{SiO}_{2}$ film of the PMOS transistor. The variations in ionization density result in different amounts of charge recombination in the track which, in turn, produces a spread in the response $\left(\Delta V_{T}\right)$ per krad.

The dose information provided by the SMD is to be used primarily to estimate the radiation damage sustained by the on-board microelectronics, and, hence, the remaining useful life of the spacecraft. A secondary objective is to provide the capability for detecting significant, shortterm increases in the intensity of the space radiation environment that can arise from natural phenomena (as for example, solar flares).

\section{RADIATION RESPONSE MEASUREMENTS}

The requirements for an MOS device that is to be used as a dosimeter are the opposite of those required for a device that is to be employed in a logic or other task-performing circuit intended for long-term use in an intense radiation environment. The dosimetric application requires the following: a reasonably high sensitivity to radiation; and an insignificant amount of annealing of the radiation damage $\left(\Delta V_{T}\right)$ with time at or near the temperatures at which the device is to be operated. Both the UK and RCA PMOS transistors satisfy these requirements very well. Additionally, samples of both devices exhibit very stable performance, that is, the initial threshold voltage fluctuations are equal to or less than 0.05 percent of $V_{T}$ over a 5 minute measurement interval. The initial threshold voltages are about 2 volts.

A typical Co- 60 calibration curve is shown in Fig. 4 for one of the RCA PMOS transistors. The dosimetric techniques employed in obtaining these results are described in an earlier publication (5). The straight line is a linear least-squares fit to the first part of the data. The sublinear response is interpreted as being due to hole-trap depletion with increasing dose. These data are obtained with the voltage applied to the gate during irradiation, $V_{G}$, being +12 volts. The possible error introduced into the measurement of dose with a PMOS device that is caused by differences in charge recombination for various radiations has been reported in reference 5. These results are summarized in Fig. 5.

Figure 5 gives the percentage of unrecombined charge in the $\mathrm{SiO}_{2}$ film of the PMOS device as a function of the gate bias, $\mathrm{V}_{G}$, for the radiations indicated. The $\mathrm{Cu} X$ rays are employed in order to simulate moderate energy protons. The details of how these curves are generated are discussed in reference 5. The important conclusion to draw from Fig. 5 is that the error introduced into the measurement of dose in a variable

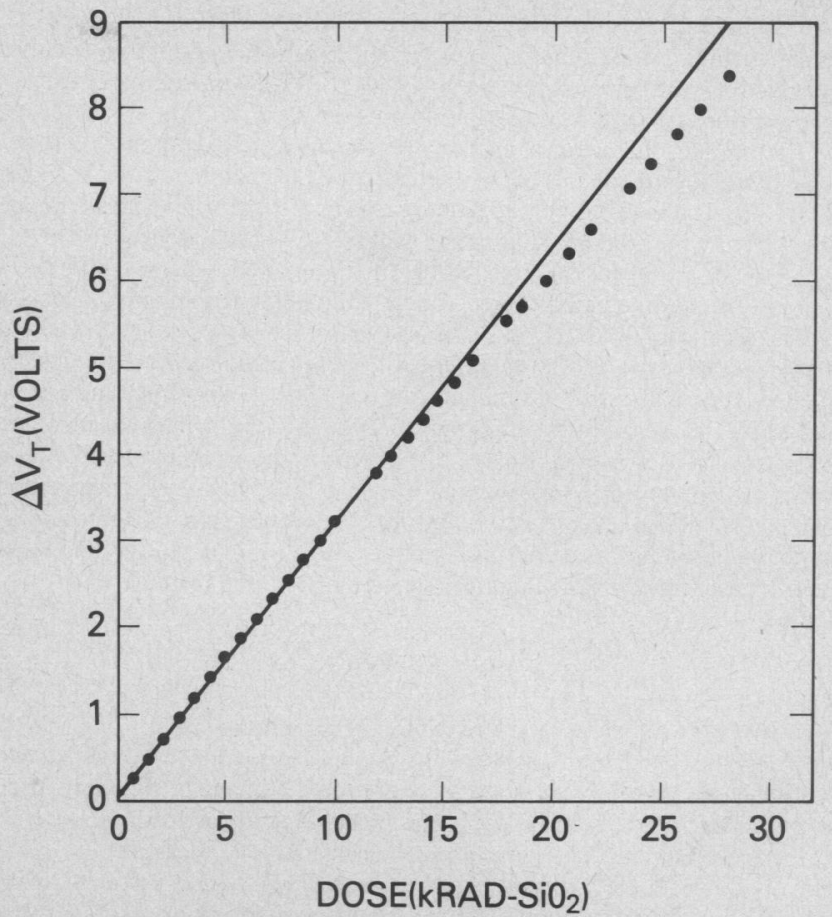

Fig. 4 - Calibration curve for an RCA PMOS transistor irradiated with Co-60 gamma rays. The straight line is a linear least-squares fit to the first 15 data points.

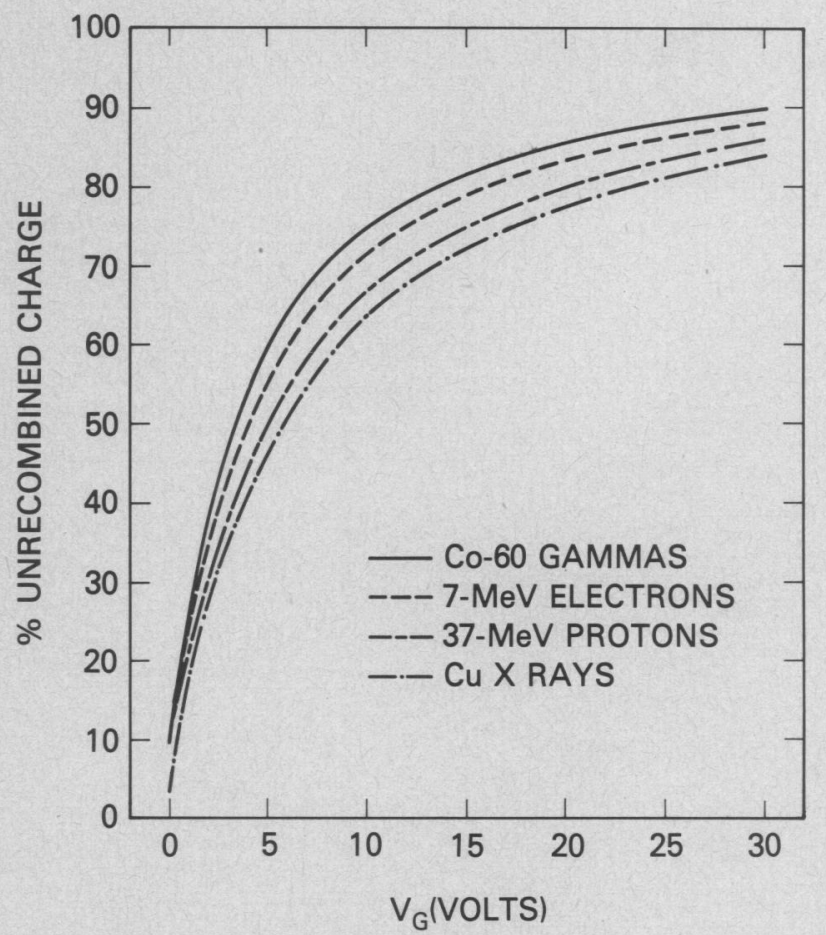

Fig. 5 - The percentage of unrecombined charge in the $\mathrm{SiO}_{2}$ film of an RCA PMOS device exposed to the indicated radiations.

radiation environment due to differences in charge recombination is greatly reduced at the higher gate biases. As a practical matter, $+10 \mathrm{~V}$ $\leqq V_{G} \leqq+20 \mathrm{~V}$ is a reasonable and attainable range of voltages over which the UK and RCA PMOS devices can be operated in order to give reliable dose measurements for the energetic electrons and protons expected in the space radiation environment. In the circuit presently employed in the SMD, $V_{G}$ is $+11.4 \mathrm{~V}$. Experience will show if a higher value would be of any significant benefit. 
Table I gives some typical data for 14 UK PMOS devices that are irradiated under conditions of approximate electronic equilibrium with a calibrated Co-60 gamma-ray source. The PMOS devices are usually exposed for 10 or 15 minute intervals at a dose rate of $23.2 \mathrm{rad}$ $\left(\mathrm{SiO}_{2}\right) /$ minute. In order to put all of the measured responses on the same basis, the threshold voltage shifts, $\Delta V_{T}$, are computed for a $1 \mathrm{krad}$ $\left(\mathrm{SiO}_{2}\right)$ dose. These computed voltage shifts are the ones shown in the table. From the voltage shift data, calibration curves are generated for each PMOS transistor by calculating the integrated voltage shifts as a function of the integrated doses. These individual calibration curves are linearly least-squares fitted in order to obtain the slopes which are taken as the best measure of the sensitivities. These calculated sensitivities are given in the right-most column in Table I. The mean sensitivity for all 14 PMOS devices is $409 \mathrm{mV} / \mathrm{krad}\left(\mathrm{SiO}_{2}\right)$, and the calculated standard deviation is $10 \mathrm{mV} / \mathrm{krad}\left(\mathrm{SiO}_{2}\right)$. In practice, the individual calibration curves are employed in determining the dose rather than the mean value. The 14 PMOS devices measured are from the same wafer. These devices are to be installed in several units of the SMD. The circuit employed in these measurements is a laboratory version of the one in the SMD.

\section{ELECTRONICS}

In Fig. 6 is shown the complete circuit for the SMD. The circuit provides the needed regulated voltages and switching to go from the expose mode to the read mode of operation. The most important parts of this circuit are those that apply the positive gate bias to the transistors in the expose mode of operation during irradiation, measure $V_{T}$ in the read mode of operation, and provide the needed analog voltages to the spacecraft telemetry system. Note that in this application what is called the threshold voltage, $V_{T}$, is really that negative gate voltage that gives a selected constant source to drain current. The partial circuit shown in Fig. 7 provides the positive gate bias in the expose mode and a measurement of $V_{T}$ in the read mode. In the expose mode, $V_{\text {reg }}$ is shorted to ground, and the offset voltage of 1.21 volts (from Zener $D_{3}$ in Fig. 6) acts through $R_{i 3}$ to cause the output of the operational amplifier A1 to saturate at a high value and thereby applies the $V_{G}=+11.4$ volts to the
TABLE I - Threshold voltage shifts in $\mathrm{mV}$ corresponding to 1 krad doses for 7 runs with 14 UK PMOS transistors. The sensitivities are the slopes of the linear least-squares fits to the $\Sigma \Delta V_{T}$ versus $\Sigma D$ results generated from the tabulated voltage shifts.

\begin{tabular}{|c|ccccccc|c|}
\hline \multirow{2}{*}{$\begin{array}{c}\text { Device } \\
\text { Number }\end{array}$} & \multicolumn{7}{|c|}{ Run Number } & Sensitivity \\
\cline { 2 - 8 } & 1 & 2 & 3 & 4 & 5 & 6 & 7 & \\
\hline \hline 6 & 396.9 & 391.1 & 391.1 & 376.8 & 385.4 & 379.6 & 374.6 & 383.0 \\
7 & 422.8 & 414.2 & 408.4 & 405.5 & 405.5 & 399.8 & 397.3 & 405.1 \\
11 & 422.8 & 418.5 & 414.2 & 405.5 & 414.2 & 405.5 & 405.5 & 410.3 \\
12 & 425.7 & 419.9 & 419.9 & 411.3 & 411.3 & 408.4 & 404.2 & 412.5 \\
15 & 422.8 & 411.3 & 408.4 & 405.5 & 399.8 & 399.8 & 397.3 & 403.5 \\
17 & 411.3 & 411.3 & 402.6 & 402.6 & 396.9 & 396.9 & 390.5 & 400.0 \\
22 & 417.0 & 411.3 & 411.3 & 402.6 & 405.5 & 402.6 & 404.2 & 405.9 \\
23 & 434.3 & 422.8 & 417.0 & 419.9 & 414.2 & 414.2 & 406.4 & 416.0 \\
25 & 425.7 & 422.8 & 417.0 & 419.9 & 411.3 & 411.3 & 408.7 & 415.1 \\
27 & 419.9 & 411.3 & 408.4 & 411.3 & 402.6 & 405.5 & 399.6 & 406.6 \\
28 & 409.8 & 409.8 & 409.8 & 409.8 & 401.2 & 401.2 & 401.2 & 405.5 \\
30 & 431.4 & 418.5 & 414.2 & 418.5 & 414.2 & 405.5 & 409.8 & 413.6 \\
31 & 431.4 & 422.8 & 427.1 & 422.8 & 418.5 & 414.2 & 418.5 & 420.7 \\
39 & 431.4 & 435.7 & 427.1 & 427.1 & 422.8 & 418.5 & 414.2 & 424.2 \\
\hline
\end{tabular}

PMOS gate. In the read mode, the current $I_{D S}$ flowing from the source to the drain of the PMOS device is regulated by the output voltage of Al which is applied to the PMOS gate. Current regulation is achieved in the following manner. The drain voltage, $V_{D}$, of the PMOS transistor is applied to the positive (noninverting) input of $\mathrm{A} 1 . V_{D}$ is given by: $V_{D}=I_{D S} R_{i 3}+1.21 \mathrm{~V}$. The output of the operational amplifier $\mathrm{Al}$ seeks a level such that $V_{D}$ is equal to the voltage at the negative (inverting) input to Al. The inverting input voltage is determined by the voltage divider made up of $R_{i 1}$ and $R_{i 2}$. The gate voltage, $V_{g}$, is related to the output of the operational amplifier A2. The output from A2, $V_{A}$, is an input to the spacecraft telemetry system. In order to determine the threshold voltage, the value of the regulated voltage, $V_{\text {reg, }}$, must be known. This voltage is determined with the partial circuit shown in Fig. 8. The output, $V_{m}$, from the LH0044AH operational amplifier is related to $V_{\text {reg. }}$.

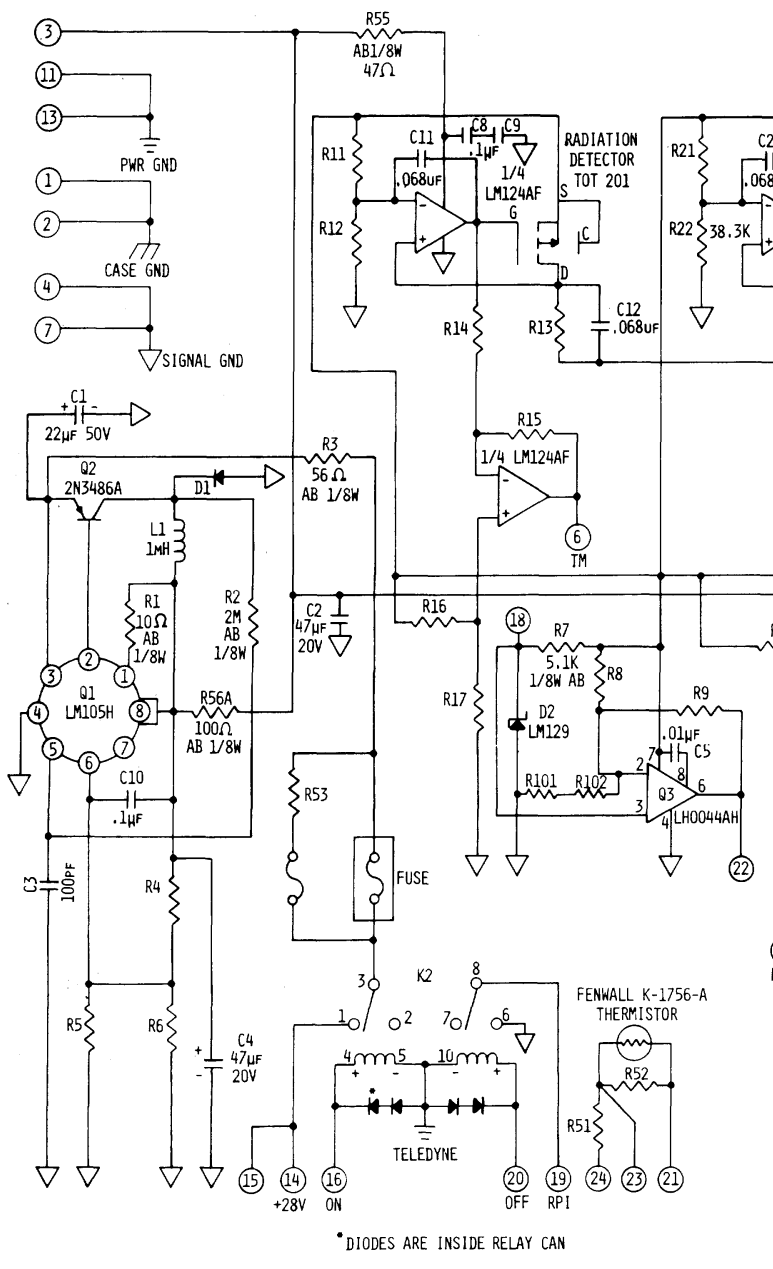

Fig. 6 - Complete diagram for the SMD circuit. Note the similarity of the partial circuits associated with each of the four PMOS transistors (designated as TOT 201s). The index $i(i=1$ to 4$)$ in the next figure is used to refer to these similar circuits for the ith PMOS transistor. 


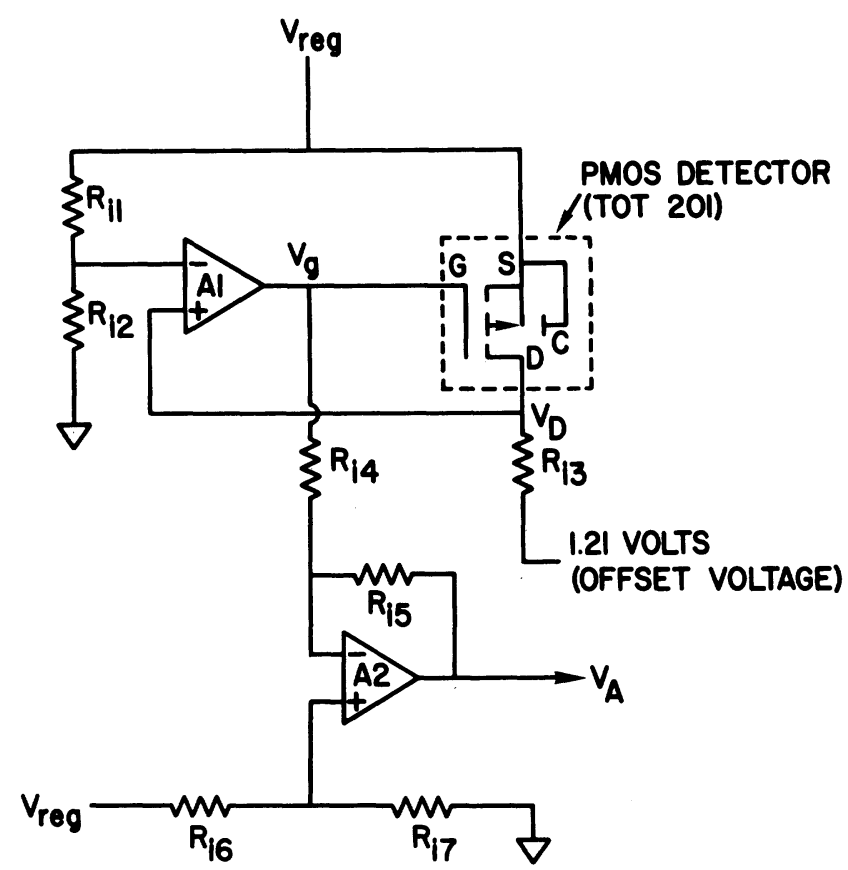

Fig. 7 - Partial circuit showing a typical arrangement for applying $V_{G}$, the gate bias during irradiation, and obtaining $V_{A}$, which is related to the threshold voltage, $V_{T}$.

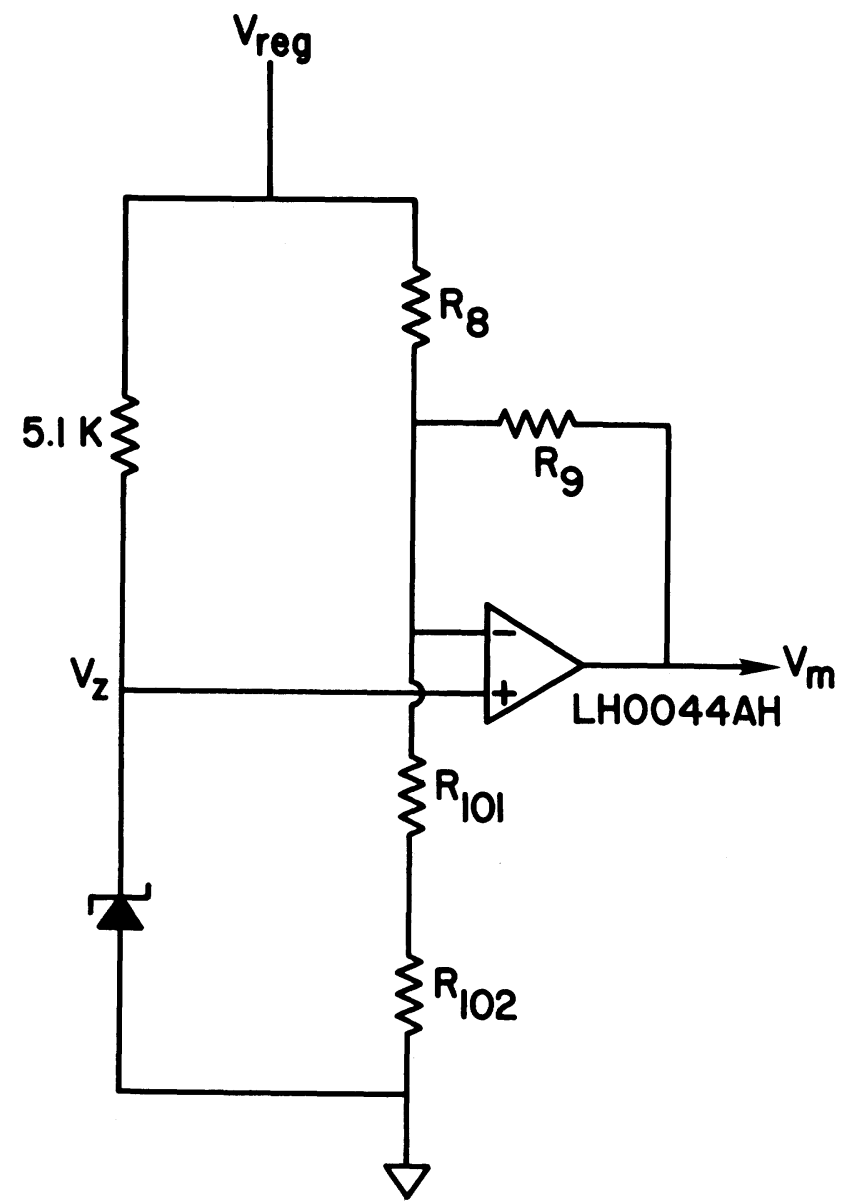

Fig. 8 - Partial circuit showing how $V_{m}$ is obtained. $V_{m}$ is related to $V_{\text {reg. }}$.
The algorithms that provide the desired voltages are:

$$
\begin{aligned}
V_{\text {reg }} & =R_{8}\left[\frac{\left(V_{z}-V_{m}\right)}{R_{9}}+\frac{V_{z}}{R_{10}}\right]+V_{z}, \\
R_{10} & =R_{101}+R_{102}, \\
V_{g} & =V_{\text {reg }} \frac{R_{i 7}}{R_{i 5}}\left[\frac{R_{i 4}+R_{i 5}}{R_{i 6}+R_{i 7}}\right]-V_{A} \frac{R_{i 4}}{R_{i 5}}, \text { and } \\
V_{T} & =V_{\text {reg }}-V_{g} .
\end{aligned}
$$

The spacecraft telemetry provides not only $V_{m}$ and $V_{A}$, but also the onoff status of the SMD, the mode (expose or read), and the temperature. The temperature is employed in order to apply small corrections to the PMOS and Zener outputs. The temperature dependence of these devices is established by extensive laboratory measurements.

The amount of aluminum shielding around the various PMOS transistors varies substantially in order to obtain data along the entire anticipated depth-dose curve. The expected doses for a given orbit are calculated from available space radiation models. Using these results the expected voltage shifts can also be computed from the measured device sensitivities. For a specific PMOS device, the gain of the operational amplifier A2 associated with it is adjusted for the predicted maximum voltage shift in order to make the optimum use of the available telemetry bits (usually 256).

The circuit shown in Fig. 6, but excluding the PMOS transistors, consists of radiation-hard components. This circuit has been tested to 150 krad with 7-MeV electrons from the Naval Research Laboratory Linac, and there was no observable degradation in performance. This dose level is typical of what would be encountered in the earth's natural radiation belts at low or moderate altitudes and substantially above what would be encountered at high altitudes.

In addition to the radiation testing discussed, the SMD is also given the usual space qualification tests, including, quality assurance of parts, shake and vibration, and temperature cycling.

\section{CONCLUSIONS}

The SMD has been tested, calibrated, and space qualified. The operation of the PMOS devices at a high positive gate bias during irradiation is expected to result in reliable dose measurements being made on the predominant radiations inside of spacecraft, namely, the energetic electrons and protons that penetrate the shielding surrounding the sensors.

\section{ACKNOWLEDGMENTS}

The authors wish to thank A. Holmes-Siedle and G. J. Brucker for helpful discussions as well as the assistance provided in acquiring the PMOS transistors. This research is funded by the Naval Electronic Systems Command, Code 615, under the NRL/NAVELEX Nuclear Survivability/Vulnerability Program.

\section{REFERENCES}

1. L. Adams and A. Holmes-Siedle, IEEE Trans. Nucl. Sci., NS-25, 1607 (1978)

2. The UK PMOS devices were obtained with the assistance of A. Holmes-Siedle.

3. The RCA PMOS devices were obtained with the assistance of G. J. Brucker.

4. A. Holmes-Siedle, Nucl. Inst. and Methods, 121, 169 (1974).

5. L.S. August, to be published in the IEEE Trans. Nucl. Sci., NS-29, Vol. 6 (1982). 